\title{
Perturbation of H3K27me3-Associated Epigenetic Processes Increases Agrobacterium-Mediated Transformation
}

\author{
Hidekazu Iwakawa, ${ }^{1}$ Benjamin C. Carter, ${ }^{2}$ Brett C. Bishop, ${ }^{2}$ Joe Ogas, ${ }^{2}$ and Stanton B. Gelvin ${ }^{1}$ \\ ${ }^{1}$ Departments of Biological Sciences; and ${ }^{2}$ Biochemistry, Purdue University, West Lafayette, IN 47907-1392, U.S.A.
}

Accepted 23 November 2016.

\begin{abstract}
Agrobacterium-mediated transformation is a core technology for basic plant science and agricultural biotechnology. Improving transformation frequency is a major goal for plant transgenesis. We previously showed that T-DNA insertions in some histone genes decreased transformation susceptibility, whereas overexpression of several Arabidopsis H2A and H4 isoforms increased transformation. Overexpression of several histone $\mathrm{H} 2 \mathrm{~B}$ and $\mathrm{H} 3$ isoforms had little effect on transformation frequency. However, overexpression of histone H3-11 (HTR11) enhanced transformation. HTR11 is a unique $\mathrm{H3}$ variant that lacks lysine at positions 9 and 27. The modification status of these lysine residues in canonical $\mathrm{H3}$ proteins plays a critical role in epigenetic determination of gene expression. We mutated histone H3-4 (HTR4), a canonical H3.3 protein that does not increase transformation when overexpressed, by replacing either or both $\mathrm{K} 9$ and $\mathrm{K} 27$ with the amino acids in HTR11 (either K9I, K27Q, or both). Overexpression of HTR4 with the K27Q but not the K9I substitution enhanced transformation. $\mathrm{HTR}^{\mathrm{K} 27 \mathrm{Q}}$ was incorporated into chromatin, and $\mathrm{HTR}^{\mathrm{K} 27 \mathrm{Q}}$ overexpression lines exhibited deregulated expression of H3K27me3enriched genes. These results demonstrate that mutation of $K 27$ in H3.3 is sufficient to perturb H3K27me3-dependent expression in plants as in animals and suggest a distinct epigenetic role for histone HTR11. Further, these observations implicate manipulation of H3K27me3-dependent gene expression as a novel strategy to increase transformation susceptibility.
\end{abstract}

Agrobacterium species transfer DNA (T-DNA) to plant cells. T-DNA associates with transferred bacterial virulence effector proteins and numerous plant proteins that help target these complexes to the nucleus and aid in T-DNA integration into the plant genome (Gelvin 2010a and b). T-DNA integration into the genome is random (Kim et al. 2007), raising the prospect that transgene integration and expression will be dependent

H. Iwakawa and Benjamin C. Carter contributed equally.

Current address for H. Iwakawa: Max Planck Institute for Plant Breeding Research, Carl-von-Linne-Weg 10, 50829 Cologne, Germany

Corresponding authors: S. B. Gelvin; E-mail: gelvin@purdue.edu; Fax: +1.765 .496 .1496 , Telephone: +1.765.494.4939; and J. Ogas; E-mail: ogas@purdue.edu; Fax: +1.765.494.7897; Telephone: +1.765.496.3969.

*The $\boldsymbol{e}$-Xtra logo stands for "electronic extra" and indicates that one supplementary figure and two supplementary tables are published online.

(c) 2017 The American Phytopathological Society on proteins that broadly affect chromatin structure, such as histones and histone-modifying enzymes. In fact, among those plant proteins that influence T-DNA integration are histones and proteins that modify chromatin structure such as histone deacetylases and histone chaperones (Crane and Gelvin 2007; Gelvin and Kim 2007; Lacroix et al. 2008; Mysore et al. 2000; Zhu et al. 2003). In particular, expression of specific classes of histones can alter transformation susceptibility of host plants. For example, the Arabidopsis rat5 mutation, an insertion into the $3^{\prime}$ untranslated region of the histone $\mathrm{H} 2 \mathrm{~A}-1$ gene HTAl, shows high levels of transient transformation (not requiring T-DNA integration) but low levels of stable transformation and T-DNA integration (Mysore et al. 2000). Indeed, the pattern of HTAI expression in roots correlates with those tissues that are most susceptible to transformation (Yi et al. 2002). Furthermore, expression of the HTAl promoter, but not promoters of other HTA genes, responds to wounding and Agrobacterium infection (Yi et al. 2006).

Overexpression of specific histones is sufficient to alter susceptibility to Agrobacterium-mediated root transformation of Arabidopsis (Tenea et al. 2009). All tested histone H2A (HTA) and histone $\mathrm{H} 4$ (HFO) cDNAs increased susceptibility when overexpressed. In contrast, all of the tested histone H2B $(H T B)$ cDNAs and most of the tested histone H3 (HTR) cDNAs did not promote transformation (Tenea et al. 2009). Specifically, overexpression of HTR4, which encodes a canonical histone H3.3 protein, did not promote transformation.

Unlike the other HTR genes, overexpression of a cDNA encoding HTR11 did increase transformation susceptibility of the derived transgenic lines (Tenea et al. 2009). HTR11 is distinguished from other Arabidopsis H3 proteins by several amino acid substitutions, including the absence of lysine residues at positions 9 and 27 (Okada et al. 2005). The modification state of these lysine residues in canonical H3 histones plays a critical role in epigenetic determination of gene expression in both plant and animal systems (Becker et al. 2016; Derkacheva and Hennig 2014; Grewal and Jia 2007; Liu et al. 2010; Loidl 2004; Simon and Kingston 2013). Whereas methylation of H3K9 is strongly associated with constitutive repression of gene expression (Becker et al. 2016; Nakayama et al. 2001), the modification status of $\mathrm{H} 3 \mathrm{~K} 27$ determines conditional expression of tissue-specific genes (Lafos et al. 2011; Mozgova et al. 2015; Pasini et al. 2010; Schwartz et al. 2010). Specifically, trimethylation of lysine 27 (H3K27me3) is associated with transcriptional repression of genes in different developmental stages or in specific tissues (Lafos et al. 2011; Mozgova et al. 2015).

Substitution of lysine 9 or lysine 27 of histone H3 for a variant amino acid can perturb epigenetic regulation and gene expression in animals (Lewis et al. 2013; Herz et al. 2014; 
Wu et al. 2012). Based on this precedent, expression of HTRI1 in Arabidopsis has the potential to alter epigenetic regulatory pathways associated with the modification status of $\mathrm{H} 3 \mathrm{~K} 9$ and $\mathrm{H} 3 \mathrm{~K} 27$ and such perturbation may, in turn, contribute to the enhanced root transformation phenotype of plants that overexpress this histone. To explore the respective contributions to transformation of the amino acid residues at positions 9 and 27 of histone $\mathrm{H} 3$, we investigated the transformation susceptibility of plants that ectopically express a canonical H3.3 histone, HTR4, in which K9, K27, or both are altered to the corresponding residue of HTR11. We found that ectopic expression of $H T R 4^{K 27 Q}$ but not HTR $4^{K 9 I}$ resulted in increased transformation susceptibility, indicating that the K27Q substitution that is found in HTR11 is sufficient to enhance transformation efficiency when present in histone H3.3. We further observed that ectopically expressed HTR 11 and $\mathrm{HTR} 4^{\mathrm{K} 27 \mathrm{Q}}$ are incorporated into chromatin and that the corresponding transgenic lines exhibit deregulation of a subset of genes that are enriched for H3K27me3. Importantly, $H T R 4^{K 27 Q}$ lines that exhibited hypersusceptibility to transformation also exhibited higher levels of H3K27me3dependent transcripts, suggesting that derepression of H3K27me3-dependent genes correlates with susceptibility to transformation of roots. These findings imply unique epigenetic attributes for the relatively uncharacterized HTR 11 histone, link altered epigenetic regulation of $\mathrm{H} 3 \mathrm{~K} 27 \mathrm{me} 3$-enriched genes to increased susceptibility to Agrobacterium-mediated transformation (AMT), and provide a rationale for future approaches to increase the susceptibility to transformation of recalcitrant species by targeting the $\mathrm{H} 3 \mathrm{~K} 27 \mathrm{me} 3$-associated epigenetic machinery.

\section{RESULTS}

\section{Overexpression of histone $\mathbf{H 3}$ proteins lacking lysine 27 stimulates AMT.}

The Arabidopsis genome comprises 15 HTR or HTR-like genes (Fig. 1A) (Okada et al. 2005). Most of the encoded proteins are similar or identical in sequence (Fig. 1B). The major exceptions are HTR11, HTR12 (which encodes the centromeric histone H3 CENH3), HTR10 (which encodes a male gametespecific H3), HTR15, and HTR7 (a likely pseudogene).

We previously showed that overexpression of cDNAs that code for canonical H3 histones such as HTRI and HTR4 did not stimulate Agrobacterium-mediated root transformation of the derived transgenic lines. However, overexpression of a HTRI1 cDNA encoding a variant $\mathrm{H} 3$ histone did increase transformation (Tenea et al. 2009). Examination of the Arabidopsis histone H3 amino acid sequences (Fig. 1B) revealed that HTR11 is a unique histone $\mathrm{H} 3$ protein in which other amino acids are substituted for the conserved lysine at positions 9 and 27. K9 and K27 can be covalently modified by several factors, and the modification states of these lysine residues are important epigenetic marks that alter chromatin structure and affect gene expression (Kuzmichev et al. 2002; Maison et al. 2002; Nakayama et al. 2001; Schubert et al. 2006; Tie et al. 2009). To determine whether modification of these lysine residues in a canonical histone $\mathrm{H} 3$ is sufficient to stimulate AMT, we replaced HTR $4{ }^{\mathrm{K} 9}, \mathrm{HTR} 4{ }^{\mathrm{K} 27}$, or both with the corresponding amino acid from the HTR11 protein.

To identify these altered HTR proteins, we tagged them at their C-termini with a triple T7 epitope tag. cDNAs encoding tagged wild-type or altered HTR4 proteins were introduced into wild-type (ecotype Ws-2) Arabidopsis plants under the control of a Cauliflower mosaic virus (CaMV) double 35S promoter and a nopaline synthase (nos) terminator (Fig. 2A). We obtained and tested numerous T2-generation transgenic lines for susceptibility to transient and stable AMT.

We define increased transformation susceptibility as greater than twice the transformation frequency as that shown by wild- type plants (Tenea et al. (2009); this study). Figure 2B and C show that only 10 to $15 \%$ of the 20 independent transgenic lines containing the tagged HTR4 gene showed increased transformation susceptibility. This result is similar to that observed by Tenea et al. (2009). When HTR4 lysines 9 and 27 were changed to isoleucine and glutamine, respectively ( $\left.\mathrm{HTR} 4{ }^{\mathrm{K} 9 \mathrm{IK} 27 \mathrm{Q}}\right)$, which are the corresponding residues present in HTR $11,21 \%$ of the 14 lines tested showed increased transformation susceptibility. This percentage of lines showing increased susceptibility mirrored that of lines overexpressing HTR11 (15 to 28\%) (Tenea et al. 2009). To determine if a single amino acid substitution for either lysine was sufficient to increase susceptibility, we generated constructions to overexpress, individually, HTR $4^{\mathrm{K} 9 \mathrm{I}}$ or HTR $4^{\mathrm{K} 27 \mathrm{Q}}$. Of the 45 or more independent lines containing the $H T R 4^{K 27 Q}$ gene, 27 to $35 \%$ showed increased transformation susceptibility. Of the 24 independent lines containing the $H T R 4^{K 9 I}$ gene, only 0 to $8 \%$ showed increased susceptibility (Fig. 2B and C). Thus, the alteration of HTR $4^{\mathrm{K} 27}$ to glutamine, the corresponding amino acid of HTR11, was sufficient to generate the transformation hypersusceptibility phenotype. Alteration of $\mathrm{HTR} 4^{\mathrm{K} 27}$ to glutamine was also likely responsible for the increased transformation phenotype observed in plants expressing HTR4 ${ }^{\mathrm{K} 9 \mathrm{IK} 27 \mathrm{Q}}$.

It is possible that the lack of transformation effect of lines containing $H T R 4^{K 9 I}$ resulted from lower levels of protein expression, but Figure 3 shows that this is not the case. Protein blot analysis, using antibodies directed against the triple T7 peptide tag on the HTR4 variants, showed that there was no correlation between the amount of transgene-encoded protein in roots of these lines and the susceptibility of these lines to AMT. For example, $H T R 4^{K 9 I} \# 4$ and $H T R 4^{K 27 Q} \# 7$ are expressed at comparable levels, and yet, only $H T R 4^{K 27 Q} \# 7$ exhibits increased transformation efficiency. Similarly, $H T R 4^{K 27 Q} \# 1$ is expressed at substantially lower levels than $H T R 4^{K 27 Q} \# 7$, yet both lines exhibit increased transformation efficiency. Comparisons such as these clearly indicate that the observed differences in transformation efficiency for the different transgenic lines are not simply the result of differences in expression of the corresponding histone proteins.

\section{The HTR4 ${ }^{\mathrm{K} 27 \mathrm{Q}}$ and HTR11 proteins are incorporated into chromatin.}

To determine if ectopically expressed histones including $\mathrm{HTR} 4{ }^{\mathrm{K} 27 \mathrm{Q}}$ are incorporated into chromatin, we used chromatin immunoprecipitation followed by quantitative polymerase chain reaction (ChIP-qPCR) from seedling root tissue, the tissue used for AMT assays, to quantify the enrichment of the T7-tagged histones at various loci. Given that we were examining incorporation of a replacement histone in which lysine 27 was altered, we examined three genes known to be enriched for the transcriptionally repressive epigenetic modification H3K27me3, namely, FUS3, $A G$, and $L E C 2$ (Goodrich et al. 1997; Makarevich et al. 2006; Schubert et al. 2006), as well as the constitutively expressed gene ACTIN7 (ACT7). We analyzed incorporation of ectopically expressed HTR11 as well as ectopically expressed $\mathrm{HTR} 4^{\mathrm{K} 27 \mathrm{Q}}$. To examine the possibility that differential incorporation of $\mathrm{HTR} 4{ }^{\mathrm{K} 27 \mathrm{Q}}$ accounted for the observed differences in transformation susceptibility, we examined incorporation of $\mathrm{HTR} 4^{\mathrm{K} 27 \mathrm{Q}}$ in two independent transgenic lines that exhibited increased susceptibility as well as two that did not. Figure 4 depicts data collected from one set of transgenic lines demonstrating that HTR 11 and HTR4 ${ }^{\mathrm{K} 27 \mathrm{Q}}$ are incorporated into chromatin at ACT7 as well as at $\mathrm{H} 3 \mathrm{~K} 27 \mathrm{me} 3$-enriched loci. Further, we observed a general trend of greater incorporation of $\mathrm{HTR} 4^{\mathrm{K} 27 \mathrm{Q}}$ in the hypersusceptible line relative to the line that was not hypersusceptible, suggesting that the extent of incorporation of $\mathrm{HTR} 4^{\mathrm{K} 27 \mathrm{Q}}$ into chromatin helps determine the hypersusceptible phenotype of these transgenic lines. Analysis 
of the second independent set of transgenic lines as a biological replicate yielded equivalent results (Supplementary Fig. S1).

\section{Expression of HTR11 or HTR4 ${ }^{\mathrm{K} 27 \mathrm{Q}}$ perturbs expression of $\mathrm{H}^{\mathrm{K} 27 \mathrm{me} 3}$-enriched genes.}

Expression of mutant $\mathrm{H} 3{ }^{\mathrm{K} 27 \mathrm{M}}$ histones in animals results in altered expression of H3K27me3-regulated loci (Herz et al. 2014; Lewis et al. 2013). We therefore examined whether plants with ectopic expression of HTR 11 or $\mathrm{HTR} 4{ }^{\mathrm{K} 27 \mathrm{Q}}$ exhibit altered expression of $\mathrm{H} 3 \mathrm{~K} 27 \mathrm{me} 3$-regulated genes in seedling roots. We assayed three independent transgenic lines for each genotype.
We used real-time qPCR (RT-qPCR) to measure the transcript levels of the genes examined in Figure 4 in addition to the well-characterized H3K27me3 target gene PHERES1 (PHE1) (Köhler et al. 2003, 2005). We observed altered expression, either increased or decreased, of the four $\mathrm{H} 3 \mathrm{~K} 27 \mathrm{me} 3$-regulated genes in the roots of HTR11 and HTR $4^{K 27 Q}$ plants relative to transgenic lines that ectopically expressed wild-type HTR4 (Fig. 5). Importantly, the transcript level of the constitutively expressed ACT7 gene was not altered in HTR 11 or $H T R 4^{K 27 Q}$ lines, even though HTR 11 and HTR $4^{\mathrm{K} 27 \mathrm{Q}}$ are incorporated at that locus, suggesting that incorporation of HTR11 and HTR4 ${ }^{\mathrm{K} 27 \mathrm{Q}}$

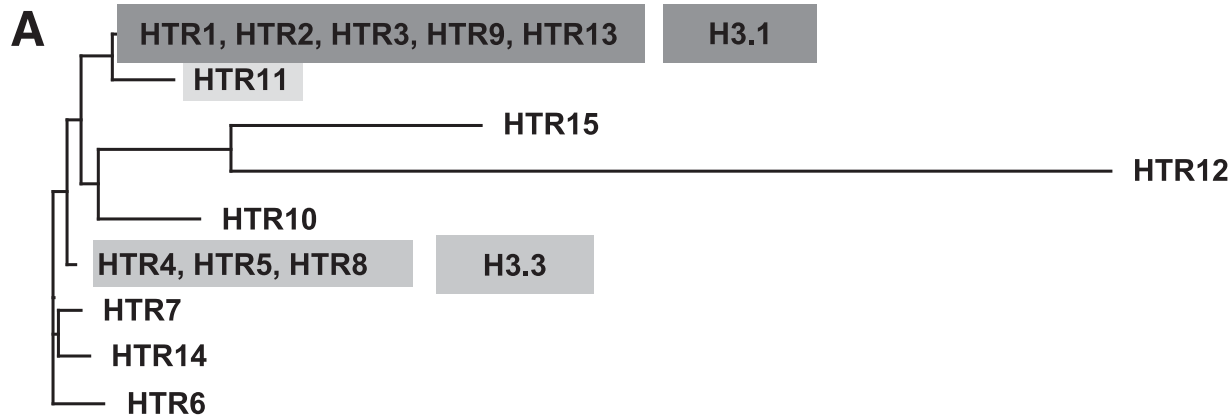

B
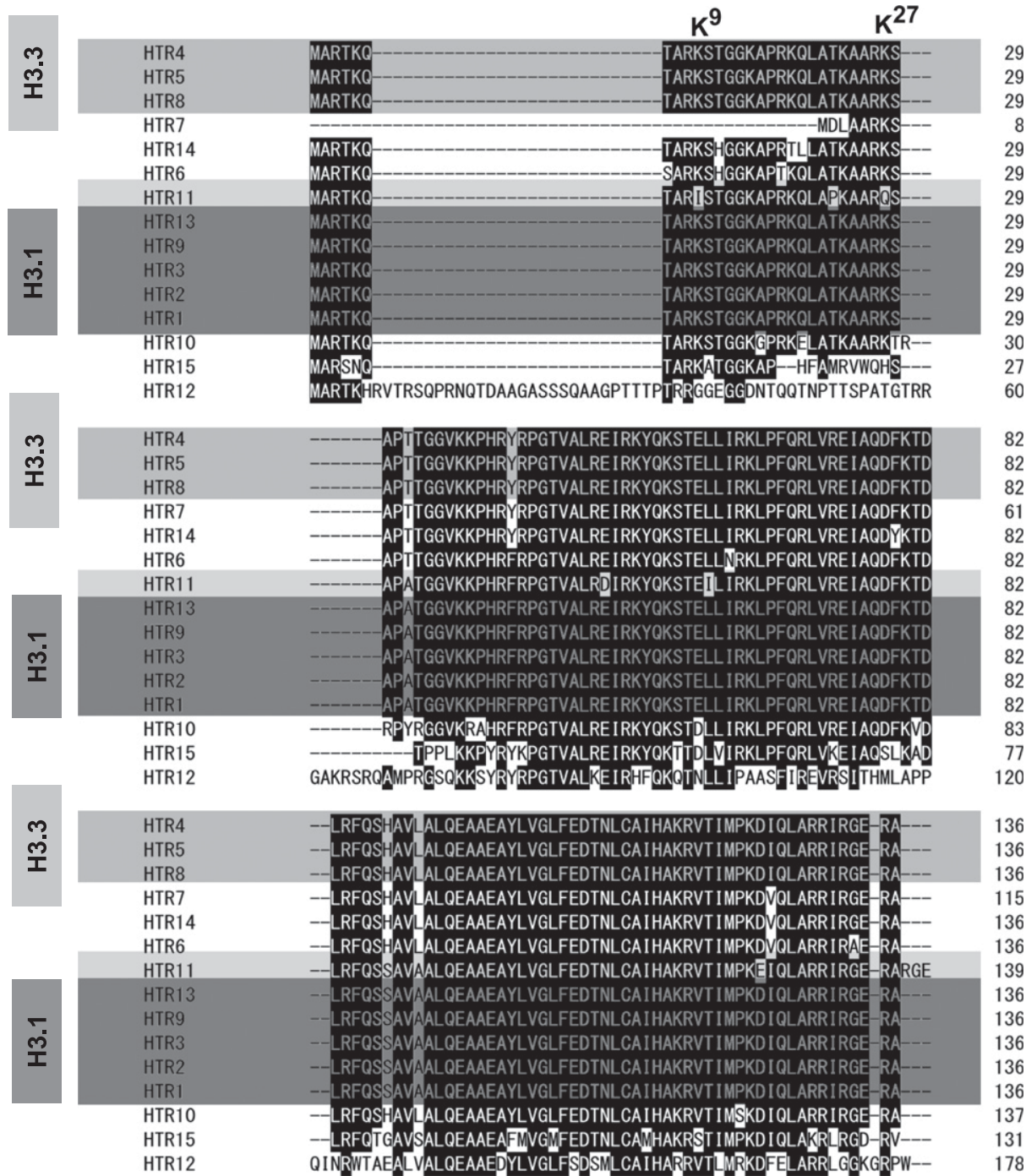

Fig. 1. A, Evolutionary relationship among Arabidopsis histone H3 proteins and B, alignment of histone H3 protein sequences. The tree was drawn by TreeView. Alignment of the protein sequences was conducted using Clustal W. Canonical H3.1 and H3.3 members are shown. 
specifically perturbs expression of genes subject to regulation by $\mathrm{H} 3 \mathrm{~K} 27 \mathrm{me} 3$. Interestingly, three $H T R 4^{K 27 Q}$ plant lines that exhibited hypersusceptibility in the transformation assays exhibited extensive derepression of the H3K27me3-dependent loci, which further correlates with the greater incorporation of the altered histone into chromatin in this line. In contrast, three nonhypersusceptible HTR 11 or HTR $4^{K 27 Q}$ lines exhibited either similar or decreased transcript levels of $A G, F U S 3$, and $L E C 1$ relative to the $A C T 7$ control gene. These data demonstrate that ectopic expression of a mutant histone $\mathrm{H} 3$ protein with an amino acid substitution for lysine 27 is sufficient to alter expression of H3K27me3-dependent loci in plants as it is in animals. Further, these data suggest that deregulation of $\mathrm{H} 3 \mathrm{~K} 27 \mathrm{me} 3$-regulated genes contributes to susceptibility to Agrobacterium transformation.

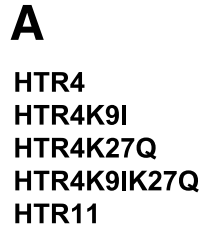

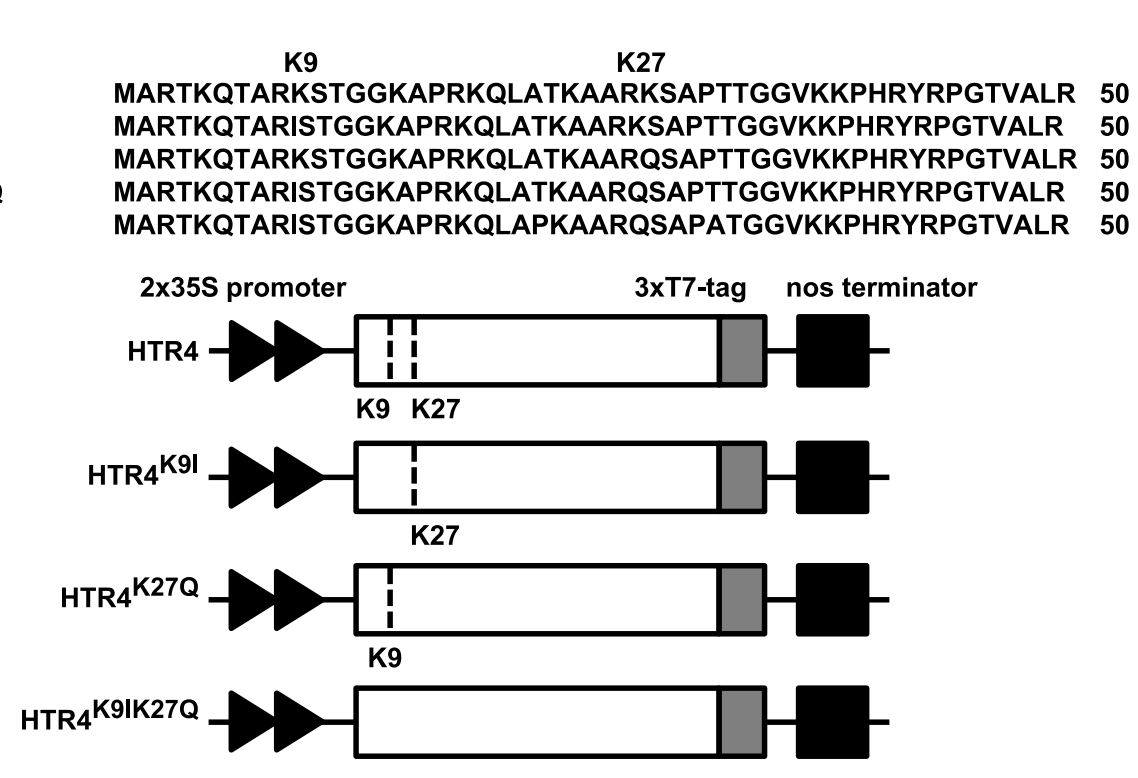

B

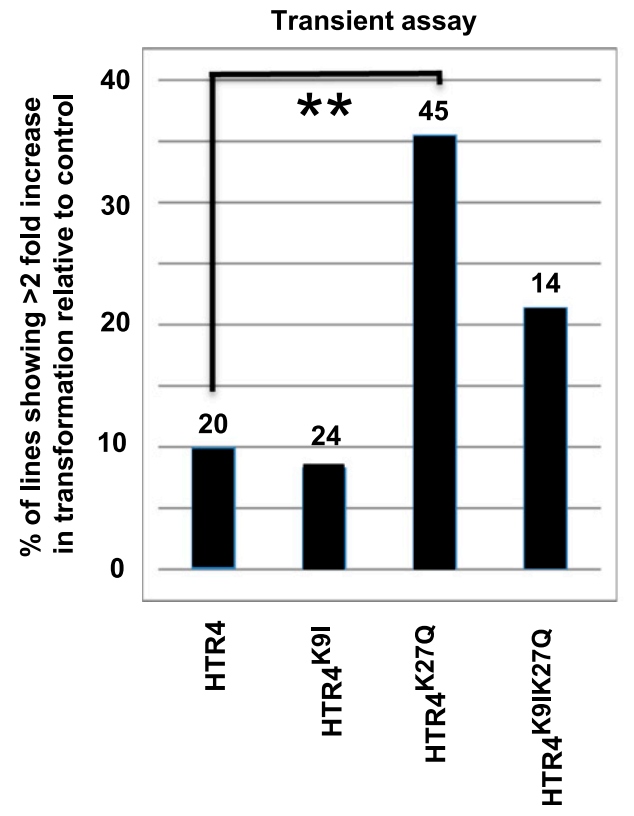

C

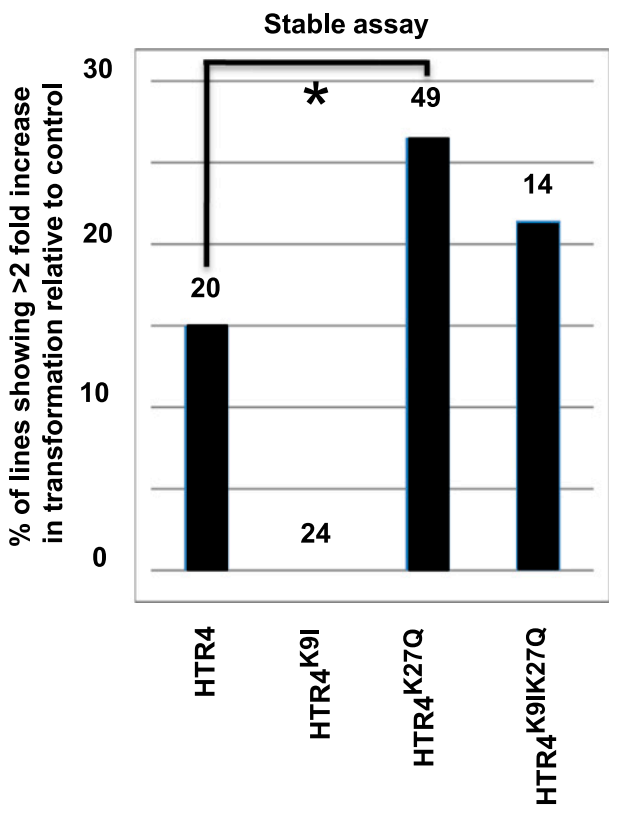

Fig. 2. Transient and stable transformation of Arabidopsis lines overexpressing HTR4 variants. A, Amino acid sequences of histone H3-11, H3-4, and H3-4 variants (top). Only the N-terminal sequences of the proteins are shown. Schematic maps of the constructs used for histone overexpression (bottom). $2 \times 35 \mathrm{~S}$ promoter $=$ Cauliflower mosaic virus double $35 \mathrm{~S}$ promoter; nos terminator $=$ nopaline synthase terminator. B, Transient transformation of T2 generation Arabidopsis transgenic lines overexpressing the indicated HTR4 or HTR4-variant cDNAs. Segments from pooled roots of five to ten plants were inoculated with Agrobacterium tumefaciens At849, containing the T-DNA binary vector pBISN1 at a concentration of $10^{6} \mathrm{CFU} / \mathrm{ml}$, and were incubated on Murashige and Skoog medium. After 2 days, the segments were moved to plates containing callus-inducing medium plus $100 \mathrm{mg}$ of timentin per milliliter and were incubated for an additional 4 days. Following X-gluc staining, the root segments were visualized using a dissecting microscope and were scored for the presence of $\beta$-glucuronidase activity. $\mathbf{C}$, Stable transformation of T2 generation Arabidopsis transgenic lines overexpressing the indicated HTR4 or HTR4 variant cDNAs. Root segments were inoculated with Agrobacterium tumefaciens A348 at a concentration of $10^{6} \mathrm{CFU} / \mathrm{ml}$ and were assayed for the formation of tumors. For B and C, numbers above the bars indicate the number of lines assayed for each construct. The frequency of obtaining high-transforming lines ( $\geq 2$-fold versus wild type) was statistically compared using onetailed exact binomial tests. Asterisks indicate $P$ values of less than $10^{-5}$ and 0.01 (B and C, respectively) for the $H T R 4^{K 27 Q}$ sample. Using a significance threshold of $\alpha=0.017$ after Bonferroni's correction, the null hypothesis of no increase in the probability of observing high-transforming lines relative to the HTR4 treatment is rejected. No statistically significant increases were observed for the $H T R 4^{K 9 I}$ or $H T R 4^{K 9 I K 27 Q}$ samples. 
Altered expression of a variety of genes by mutation or by overexpression is known to affect susceptibility to transformation by Agrobacterium spp. (Anand et al. 2007; Crane and Gelvin 2007; Hwang and Gelvin 2004; Sardesai et al. 2013; Tenea et al. 2009). Several of these genes are known to be enriched for H3K27me3 in seedlings (Table 1), raising the prospect that altered expression of these specific genes in transgenic lines expressing HTR 11 and $H T R 4^{K 27 Q}$ contributed to the observed transformation phenotypes. We tested this hypothesis by using RT-qPCR to determine whether the roots of HTR 11 and HTR4 ${ }^{K 27 Q}$ plants exhibit altered transcript levels of these genes, both those known to be enriched for H3K27me3 and those that are not. Although most of the genes did not exhibit altered transcript levels in the transgenic lines (Table 1), two of these genes did (Fig. 6), namely, the putative transmembrane receptor AT14A (Sardesai et al. 2013) and LIGHT SENSITIVE HYPOTCOTYLS 10 (LSH10) (Y. Wang, L.-Y. Lee, and S. B. Gelvin unpublished data). Both of these genes are known to promote transformation susceptibility when overexpressed and were found to exhibit altered transcript levels in lines that ectopically expressed HTR 11 and $H T R 4^{K 27 Q}$. Importantly, however, expression of AT14A or $L S H 10$ alone does not appear to be sufficient to account for the observed transformation phenotype of the lines characterized here, as increased expression of either locus does not perfectly correlate with the transformation phenotype of these lines. For example, the transcript level of $A T 14 A$ is increased in the $H T R 4^{K 27 Q}$ hypersusceptible line but not the HTR11 line (Fig. 6). However, although expression of $\mathrm{LSH1O}$ is increased in the hypersusceptible HTR11 line, it is also increased in the HTR ${ }^{K 27 Q}$ line that is not hypersusceptible. These data raise the prospect that the transformation hypersusceptible phenotype of these transgenic lines results from broad misregulation of H3K27me3-responsive loci rather than from specific misregulation of a particular locus.

\section{DISCUSSION}

These studies identify a novel connection between the H3K27me3 epigenetic pathway and Agrobacterium-mediated plant genetic transformation. It was previously shown that overexpression of the novel histone $\mathrm{H} 3$ variant encoded by HTR 11 but not canonical H3 histones resulted in a substantial increase in transformation susceptibility (Tenea et al. 2009). HTR11 is unusual among H3 proteins in that it lacks highly conserved lysine residues at positions 9 and 27. The modification state of either or both $\mathrm{K} 9$ and $\mathrm{K} 27$ plays a significant role
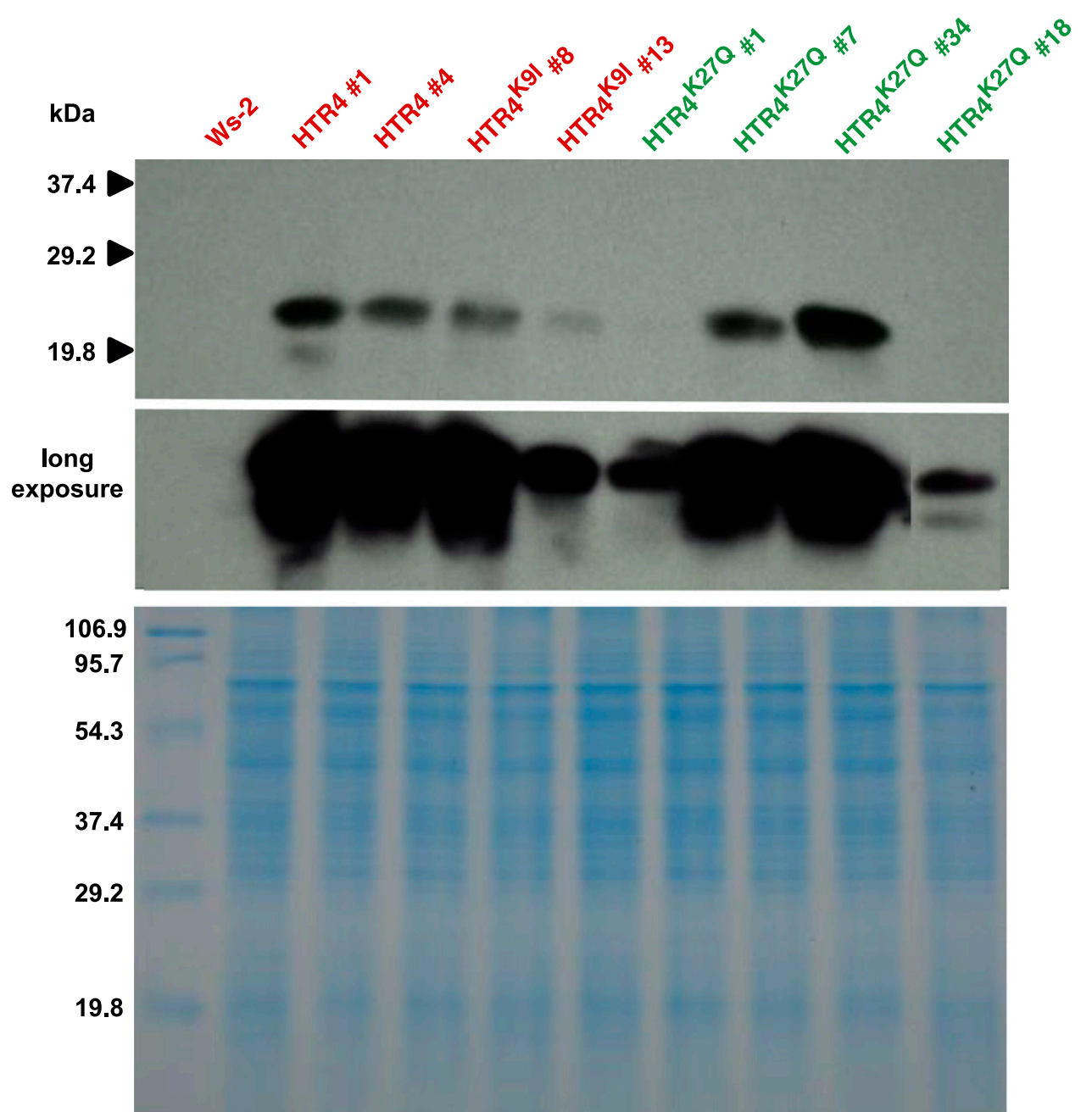

Fig. 3. Protein blot analysis of tagged histone $\mathrm{H} 3$ variants expressed in transgenic Arabidopsis roots. Proteins were extracted from roots of the indicated plant lines, were subjected to electrophoresis through sodium dodecyl sulfate polyacrylamide gels, and were transferred to a polyvinylidene difluoride membrane. The membrane was reacted with an anti-T7-Tag antibody horseradish peroxidase conjugate and was visualized using Western blotting luminol reagent. The top and middle panels show short and long exposures, respectively. The bottom panel shows a photograph of a Coomassie brilliant blue-stained gel. Numbers on the left indicate molecular size standards. The five lanes on the left did not show an increase in transformation; the four lanes on the right showed an increase in transformation. 
in epigenetic determination of gene expression in eukaryotes (Becker et al. 2016; Derkacheva and Hennig 2014; Grewal and Jia 2007; Liu et al. 2010; Loidl 2004; Simon and Kingston 2013). Our studies demonstrate that overexpression of a canonical H3 protein that has been made more "HTR11-like" by the single amino acid substitution K27Q (HTR4 ${ }^{\mathrm{K} 27 \mathrm{Q}}$ ) is sufficient to increase transformation susceptibility (Figs. 2 and 3 ). ChIP analysis revealed that both HTR11 and HTR4 ${ }^{\mathrm{K} 27 \mathrm{Q}}$ are incorporated into the chromatin of both an actively transcribed gene as well as a number of loci known to be subject to the repressive epigenetic modification H3K27me3 (Fig. 4) (Makarevich et al. 2006; Schubert et al. 2006). These same ChIP data also revealed a general trend in which hypersusceptible lines had higher levels of $\mathrm{HTR} 4^{\mathrm{K} 27 \mathrm{Q}}$ at examined loci, relative to nonhypersusceptible lines, suggesting that a minimum level of $\mathrm{HTR} 4^{\mathrm{K} 27 \mathrm{Q}}$ incorporation is necessary to promote elevated susceptibility to transformation. Importantly, the level of HTR 11 incorporation in a hypersusceptible line is comparable to the level of HTR $4{ }^{\mathrm{K} 27 \mathrm{Q}}$ incorporation in a transgenic line that is not hypersusceptible, suggesting that the presence of multiple noncanonical amino acids in HTR11 may enhance its ability to promote a hypersusceptible phenotype. Further, plants that overexpress HTR 11 or HTR4 ${ }^{\mathrm{K} 27 \mathrm{Q}}$ exhibit misregulation of H3K27me3dependent genes (Fig. 5), suggesting that incorporation of either of these histone variants into chromatin perturbs epigenetic regulation.
Intriguingly, altered gene expression was greater in lines that were hypersusceptible to transformation, suggesting that misregulation of H3K27me3-dependent genes correlates with transformation susceptibility.

$\mathrm{H} 3^{\mathrm{K} 27 \mathrm{Q}}$ is commonly regarded as a mimic for acetylated H3K27 (H3K27Ac). Glutamine structurally resembles acetylated lysine and has been used as a surrogate for this modification in vitro and in vivo (Hecht et al. 1995; Li et al. 2002). $\mathrm{H} 3 \mathrm{~K} 27 \mathrm{Ac}$ is associated with active chromatin and is also present at the promoter of $\mathrm{H} 3 \mathrm{~K} 27$ me3-dependent genes when they are transcribed (Karantzali et al. 2008; Pasini et al. 2010; Schwartz et al. 2010). Thus, the increased transcript levels of H3K27me3-dependent genes in the $H T R 4^{K 27 Q}$ lines (Fig. 5) may result from the presence of $H T R 4^{K 27 Q}$ in the promoter of these genes, which facilitates an active transcriptional state. The simultaneous observation of decreased transcript levels in HTR 11 lines as well as $H T R 4^{K 27 Q}$ lines that are not hypersusceptible, however, suggests that the impact of these altered histones on chromatin structure is likely more complex than simply mimicking the acetylated state. Future characterization of the epigenetic status of the affected genes in the overexpression lines may clarify how these modified histones are altering gene expression.

Our studies raise the possibility that the unique $\mathrm{H} 3$ variant HTR11 plays a distinct epigenetic role during development in
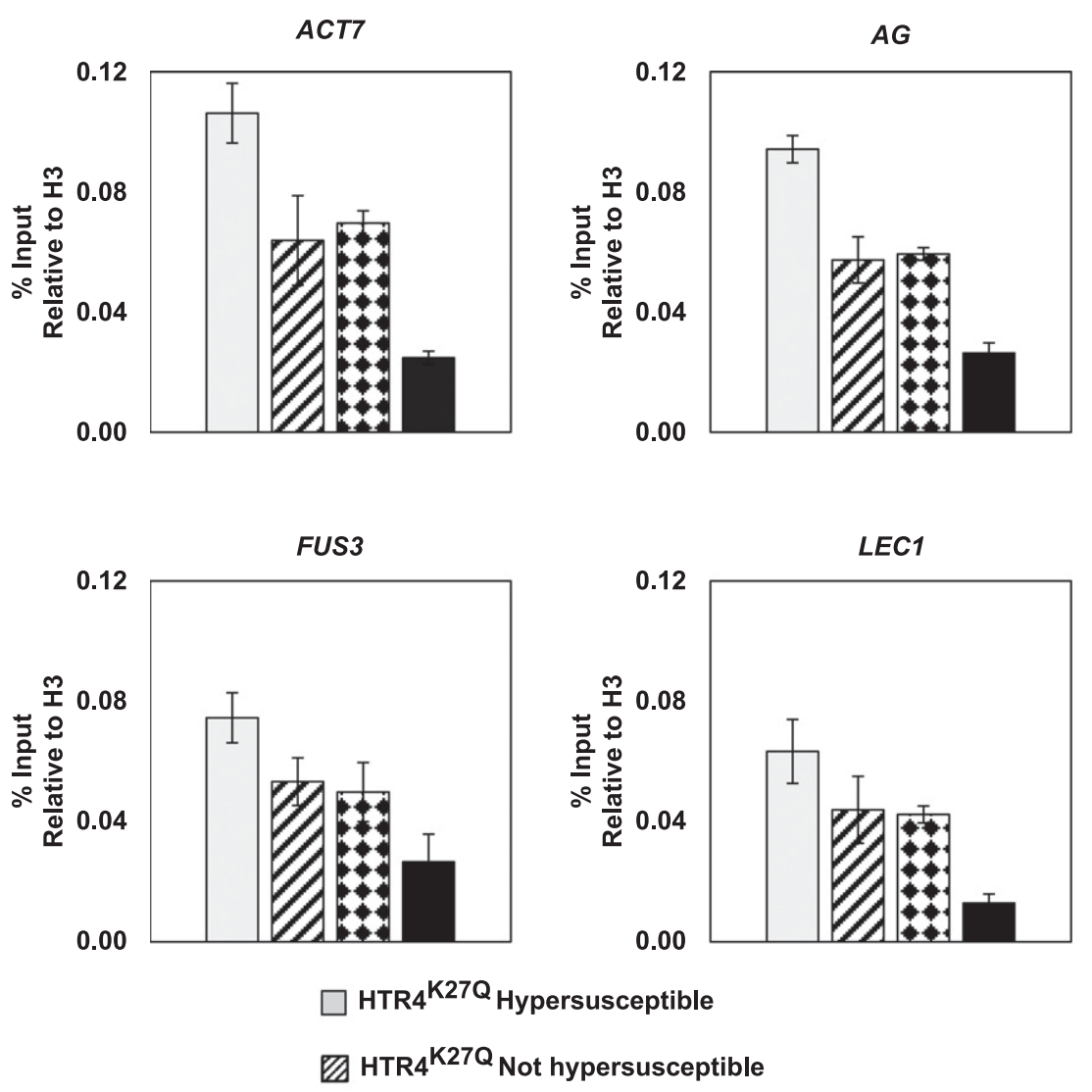

HTR11

Ws-2

Fig. 4. HTR11 and HTR4K27Q proteins are integrated into chromatin. Chromatin immunoprecipitation was used to examine the presence of the T7-tagged histone constructs at the indicated loci in seedling roots of the following genetic backgrounds: wild-type ecotype Wassilewskija (Ws-2), HTR11-overexpressing hypersusceptible plants, $H T R 4^{K 27 Q}$-overexpressing hypersusceptible plants, and $H T R 4^{K 27 Q}$-overexpressing plants that were not hypersusceptible to Agrobacterium-mediated transformation. The $y$ axis denotes percent of input DNA immunoprecipitated using an anti-T7 antibody and is normalized to H3 occupancy. All data are the average of two technical replicates of a single set of transgenic lines. Error bars indicate standard deviation of the two technical replicates. 
Arabidopsis. Although it has been suggested that full-length HTR 11 transcript is not expressed due to the presence of an intron containing the HTR11 start codon (Okada et al. 2005), RNA-seq and microarray analyses reveal that the full-length open reading frame is, in fact, expressed in the carpel tissues of flowers (Martínez-Fernández et al. 2014; Schmid et al. 2005). Indeed, we were able to obtain a HTR 11 cDNA clone from whole plant tissue. This expression raises the possibility that HTR 11 plays a role in epigenetic regulation of gene expression in Arabidopsis. Given the unique substitutions at K9 and K27, characterization of the contribution of this histone variant to chromatin structure and gene expression is of substantial interest. However, investigation of the biological role of HTR11 is complicated by its presence in a bicistronic transcript as a second open reading frame that is preceded by the histone $\mathrm{H} 3.1$ gene At5g65360 (Okada et al. 2005). Application of recently developed CRISPR (clustered regularly interspaced short palindromic repeat)-based approaches (Eid et al. 2016; Gao et al. 2016; Zhang et al. 2016) will likely be necessary to ablate selectively expression of this gene and thereby examine its potential contribution to plant development.

The observation that overexpression of $H T R 4^{K 27 Q}$ is sufficient to increase susceptibility to AMT suggests that other treatments perturbing the modification state of H3K27 may similarly alter susceptibility to transformation. Multiple compounds are available that alter levels of histone methylation or acetylation in plants (Zhang et al. 2013). Due to the structural similarities between glutamine and acetylated lysine, histone deacetylase inhibitors may be of particular interest for designing novel strategies to boost the efficiency of plant transformation by Agrobacterium tumefaciens.

\section{MATERIALS AND METHODS}

\section{Construction of plasmids.}

HTR4 and HTR 11 cDNAs were obtained as plasmids E2817 and E2819, respectively (Tenea et al. 2009). To construct plasmids for transient assays, histone cDNAs were cloned into pRTL2 (Restrepo et al. 1990) under the control of a CaMV double $35 \mathrm{~S}$ promoter. EcoRI/XbaI fragments from E2817 and E2819 were cloned into the EcoRI/XbaI sites of pBluescript $\mathrm{SK}(+)$ to generate E3903 and E3904, respectively. Synthetic nucleotides (BamXbaT7Spe-1 and BamXbaT7Spe-2) that encode a triple T7 epitope tag were cloned into the BamHI/SpeI sites of pRTL2-GUS ( $\beta$-glucuronidase) (Restrepo et al. 1990) to generate E3905. BamXbaT7T7Spe-1 and BamXbaT7T7Spe-2 were cloned into the BamHI/SpeI sites of E3905 to generate E3906. cDNAs of HTR4 and HTR11 were amplified using primers T3/HTR4rev and T3/HTR11rev, respectively. Amplified cDNAs were digested by EcoRI/XbaI and were inserted into the EcoRI/XbaI sites of E3906 to generate E3907 and E3911. For introducing the HTR4K9I or HTR4K27Q mutations, the HTR 4 cDNA was amplified using primers containing the corresponding mutation. To generate $H 3^{K 9 I}$, the $5^{\prime}$ and $3^{\prime}$ halves of HTR4 were amplified using primers T3/HTR4K9I-1 and HTR4K9I-2/HTR4rev, respectively. Amplified fragments were mixed, denatured, and annealed for the preparation of template for a second round of PCR. The HTR $4^{K 9 I} \mathrm{cDNA}$ was amplified using T3/HTR4rev primers. The amplified fragment was digested with EcoRI/XbaI and was inserted into the EcoRI/XbaI sites of E3906 to generate E3908. The HTR ${ }^{K 27 Q}$ cDNA was amplified, as described above, with the primers T3/HTR4K27Q-1 and HTR4K27Q-2/HTR4rev for the first round of PCR and with T3/HTR4rev primers for the second round of PCR. To construct the $H T R 4^{K 9 I K 27 Q}$ cDNA, the $\mathrm{K} 27 \mathrm{Q}$ mutation was introduced into $H T R 4^{K 9 I}$, as described above. Amplified DNA fragments corresponding to $H T R 4^{K 9 I}$ and HTR $4^{K 9 I K 27 Q}$ were digested with EcoRI/XbaI and were

Table 1. H3K27me3 status of genes examined using quantitative real-time polymerase chain reaction (qRT-PCR)

\begin{tabular}{lll}
\hline Locus ID & H3K27me3 $^{\mathbf{a}}$ & Transcript level $^{\mathbf{b}}$ \\
\hline At5g09810 & No & Not altered \\
At4g05320 & No & Not altered \\
At4g18960 & Zhang & Altered \\
At4g22950 & Zhang & Not altered \\
At5g38110 & Charron & Not altered \\
At3g28290 & No & Altered \\
At4g23630 & No & Not altered \\
At3g26790 & Zhang & Altered \\
At1g21970 & Zhang & Altered \\
At2g31160 & Zhang & Not altered \\
At2g42610 & Zhang & Altered \\
At2g40970 & Charron & Not altered \\
At1g65330 & Zhang & Altered \\
\hline
\end{tabular}

a No indicates that the gene is not known to be enriched in seedlings, Zhang indicates enrichment based on Zhang et al. (2007), and Charron indicates enrichment based on Charron et al. (2009) for the cases in which there is no enrichment based on Zhang et al. (2007).

b Indicates whether expression was observed to be altered in either or both HTR 11 and $H T R 4^{K 27 Q}$ samples in qRT-PCR experiments.

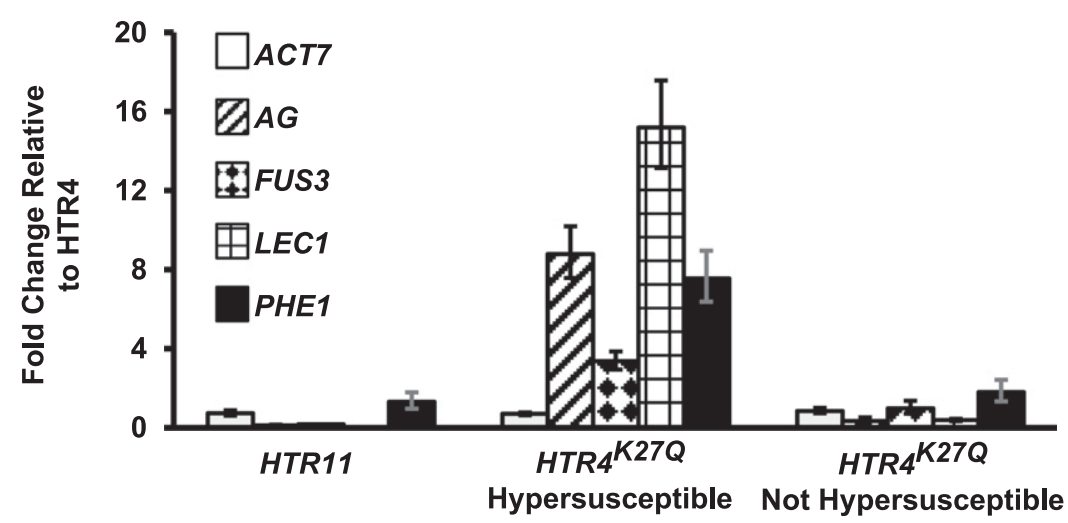

Fig. 5. Expression of H3K27me3-regulated genes is altered in the roots of HTR4K27Q and HTR 11 plants. RNA was extracted from the roots of plants overexpressing one of the following constructs: HTR $4, H T R 11$, or $H T R 4^{K 27 Q}$. HTR $4^{K 27 Q}$ samples were collected both from lines that exhibited increased susceptibility to transformation by Agrobacterium (hypersusceptible) and from lines that did not (not hypersusceptible). Data are the average of two technical replicates collected from three independent lines. Quantitative real-time polymerase chain reaction was performed to measure transcript levels of the constitutively expressed gene ACT7 as well as the indicated H3K27me3-dependent genes. 18S rRNA was used as the reference gene, and transcript levels are normalized to expression in HTR4 roots. Error bars represent standard deviation between three technical replicates. 
inserted into the EcoRI/XbaI sites of E3906, to generate E3909 and E3910, respectively. For stable transformation assays, DNA fragments including the $35 \mathrm{~S}$ promoter, the histone cDNA, and the nos terminator from E3907, E3908, E3909, or E3910, which were digested by HindIII and blunted, were cloned into the SmaI site of the T-DNA binary vector pCB302 (Xiang et al. 1999), to generate E4192, E4193, E4194, and E4195, respectively. PstI fragments containing the $35 \mathrm{~S}$ promoter, HTR 11T7, and the nos terminator from E3911 were cloned into the PstI site of pCB302 to generate E4196.

\section{Floral-dip transformation.}

Transgenic plants were generated by a flower-dip transformation protocol (Clough and Bent 1998). Floral buds of Arabidopsis ecotype Ws-2 were used for transformation with A. tumefaciens EHA105 (Hood et al. 1993) containing various T-DNA binary plasmids. Transformants were selected on Murashige and Skoog (MS) medium (Murashige and Skoog 1962) containing 2\% sucrose, $10 \mathrm{mg}$ of phosphinothricin per liter, and $100 \mathrm{mg}$ of timentin per liter.

\section{Transient and stable AMT assays.}

We conducted AMT assays as described previously (Mysore et al. 2000; Nam et al. 1999; Yi et al. 2002), with the exception described below. In brief, Arabidopsis plants, initially selected on MS medium containing phosphinothricin and timentin, were grown in baby food jars containing MS medium for 2 to 3 weeks at $25^{\circ} \mathrm{C}$. Root segments ( 2 to $5 \mathrm{~mm}$ ) were infected for 2 days with Agrobacterium tumefaciens At849 or A208 for transient or stable assays, respectively (Zhu et al. 2003). The infecting bacterial concentration was either $10^{5}$ or $10^{6} \mathrm{CFU}$ per milliliter. For the transient AMT assay, after cocultivation for 2 days, root segments were washed and cultured on MS medium containing (per liter) $5 \mathrm{mg}$ of indole acetic acid, $0.5 \mathrm{mg}$ of 2,4-dichlorophenoxyacetic acid, $0.3 \mathrm{mg}$ of kinetin, and $100 \mathrm{mg}$ of timentin. After 4 days of further cultivation, histochemical staining was performed according to Jefferson et al. (1987), with modifications. Root segments were incubated in GUS

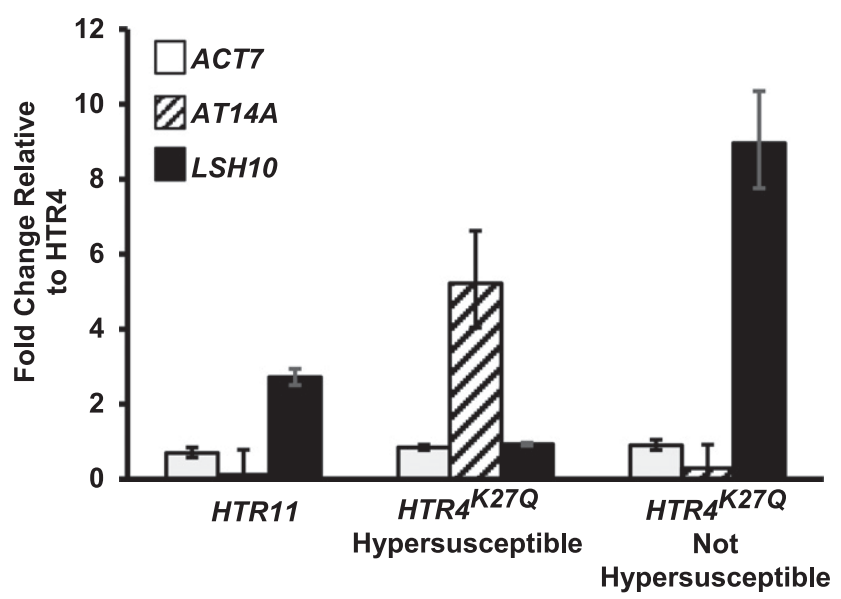

Fig. 6. Expression of $A T 14 A$ and $L S H 10$ is altered in the roots of HTR4K27Q and HTR11 plants. RNA was extracted from the roots of plants overexpressing one of the following constructs: HTR4, HTR11, or HTR4 ${ }^{K 27 Q}$. $H T R 4^{K 27 Q}$ samples were collected both from lines that exhibited increased susceptibility to transformation by Agrobacterium tumefaciens (hypersusceptible") and from lines that did not (not hypersusceptible). Quantitative real-time polymerase chain reaction was performed to measure transcript levels of the constitutively expressed gene ACT7 as well as AT14A and LSH10. 18S rRNA was used as the reference gene and transcript levels are normalized to expression in HTR4 roots. Data are representative of three biological replicates. Error bars represent standard deviation between three technical replicates. staining solution ( $2 \mathrm{mM}$ 5-bromo-4-chloro-3-indolyl- $\beta$-Dglucoronide [X-gluc], $100 \mathrm{mM}$ sodium phosphate, $\mathrm{pH} 7.0$, $\left.0.1 \mathrm{mM} \mathrm{K}_{3}\left[\mathrm{Fe}\left(\mathrm{CN}_{6}\right)\right], 0.1 \mathrm{mM} \mathrm{K}_{4}\left[\mathrm{Fe}\left(\mathrm{CN}_{6}\right)\right]\right)$ at $37^{\circ} \mathrm{C}$ overnight. Transient transformation was scored as the percentage of root segments staining blue. For stable AMT, after cocultivation for 2 days, root segments were washed and separated onto solidifed MS medium containing $100 \mathrm{mg}$ of timentin per liter. Tumors were scored 3 to 4 weeks later.

\section{Immunoblot analysis.}

Proteins were extracted from Arabidopsis roots into sample buffer (62.5 mM Tris [pH6.8], 2\% sodium dodecyl sulfate [SDS], 5\% glycerol, $0.005 \%$ bromophenol blue, and $0.1 \mathrm{M}$ 2-mercaptoethanol), from plants selected on MS medium containing phosphinothricin and timentin, and were grown in baby food jars containing MS medium for 3 weeks at $25^{\circ} \mathrm{C}$. Cell debris was removed by centrifugation and the supernatant solution was used. A total of $200 \mu \mathrm{g}$ of proteins were boiled for $10 \mathrm{~min}$ and were subjected to SDSpolyacrylamide gel electrophoresis through a $12.5 \%$ polyacrylamide gel. Proteins were transferred to a Hybond-P polyvinylidene difluoride membrane (Amersham Biosciences) in transfer buffer (10 mM Tris-HCl, 192 mM glycine, 20\% methanol). The membrane was blocked overnight at room temperature in 5\% skimmed milk dissolved in Tris-buffered saline containing $0.1 \%$ Tween-20 (TBS-T), was washed twice in TBS-T, was incubated with a T7-Tag antibody horseradish peroxidase conjugate (Novagen), and was visualized using Western blotting luminol reagent (Santa Cruz Biotechnology).

\section{Multiple alignment and construction of trees.}

Sequences of 13 histone $\mathrm{H} 3 \mathrm{~s}$ were obtained from the TAIR10 database. Alignment and a tree of all Arabidopsis histone H3 DNA sequences were constructed using Clustal W (Thompson et al. 1994). The tree was drawn by TreeView (Page 1996).

\section{RNA isolation and analysis.}

Total RNA was isolated using a Qiagen RNeasy plant mini kit from the roots of 3-week-old wild-type Ws-2 plants as well as Ws-2 plants overexpressing the following constructs: HTR4, HTR 1 1, HTR $4^{K 27 Q}$ (hypersusceptible plants), and HTR $4^{K 27 Q}$ (not hypersusceptible plants). The RNA samples were DNasetreated and were concentrated using the Zymo Research RNA clean and concentrator kit. First-strand cDNA synthesis and qRT-PCR were performed as described previously (Carter et al. 2016). Oligonucleotide primers used for qRT-PCR analyses are described in Supplementary Table S2.

\section{ChIP analysis.}

ChIP was performed as previously described (Zhang et al. 2012), with the following modifications. Tissue (3 g of 3-weekold roots) was harvested from plants of the genotypes listed above. Chromatin was sheared into approximately 250- to 750bp fragments by sonication, using a Covaris E220 sonicator. The samples were diluted threefold in ChIP dilution buffer $(1.1 \%$ Triton X-100, 1.2 mM EDTA, $16.7 \mathrm{mM}$ Tris-HCl, pH 8.0, and $167 \mathrm{mM} \mathrm{NaCl})$. Supernatant solution $(500 \mu \mathrm{l})$ was used for each immunoprecipitation. Millipore anti-T7 antibody was added at 1: 100 dilution and the tubes were rotated at $4{ }^{\circ} \mathrm{C}$ for about $15 \mathrm{~h}$. Dynabeads Protein G (50 $\mu \mathrm{l})$ (Invitrogen) were equilibrated with ChIP dilution buffer (described above) as a 50\% slurry and were added to each tube.

\section{ACKNOWLEDGMENTS}

This work was funded by grants from the National Science Foundation to S. B. Gelvin and to J. Ogas. 


\section{LITERATURE CITED}

Anand, A., Krichevsky, A., Schornack, S., Lahaye, T., Tzfira, T., Tang, Y., Citovsky, V., and Mysore, K. S. 2007. Arabidopsis VIRE2 INTERACTING PROTEIN2 is required for Agrobacterium T-DNA integration in plants. Plant Cell 19:1695-1708.

Becker, J. S., Nicetto, D., and Zaret, K. S. 2016. H3K9me3-dependent heterochromatin: Barrier to cell fate changes. Trends Genet. 32: $29-41$.

Carter, B., Henderson, J. T., Svedin, E., Fiers, M., McCarthy, K., Smith, A., Guo, C., Bishop, B., Zhang, H., Riksen, T., Shockley, A., Dilkes, B. P., Boutillier, K., and Ogas, J. 2016. Cross-talk between sporophyte and gametophyte generations is promoted by $\mathrm{CHD} 3$ chromatin remodelers in Arabidopsis thaliana. Genetics 203:817-829.

Charron, J. B., He, H., Elling, A. A., and Deng, X. W. 2009. Dynamic landscapes of four histone modifications during deetiolation in Arabidopsis. Plant Cell 21:3732-3748.

Clough, S. J., and Bent, A. F. 1998. Floral dip: A simplified method for Agrobacterium-mediated transformation of Arabidopsis thaliana. Plant J. 16:735-743.

Crane, Y. M., and Gelvin, S. B. 2007. RNAi-mediated gene silencing reveals involvement of Arabidopsis chromatin-related genes in Agrobacteriummediated root transformation. Proc. Natl. Acad. Sci. U.S.A. 104 15156-15161.

Derkacheva, M., and Hennig, L. 2014. Variations on a theme: Polycomb group proteins in plants. J. Exp. Bot. 65:2769-2784

Eid, A., Ali, Z., and Mahfouz, M. M. 2016. High efficiency of targeted mutagenesis in Arabidopsis via meiotic promoter-driven expression of Cas9 endonuclease. Plant Cell Rep. 35:1555-1558.

Gao, X., Chen, J., Dai, X., Zhang, D., and Zhao, Y. 2016. An effective strategy for reliably isolating heritable and Cas9-free Arabidopsis mutants generated by CRISPR/Cas9-mediated genome editing. Plant Physiol. doi:10.1104/pp.16.00663

Gelvin, S. B. 2010a. Finding a way to the nucleus. Curr. Opin. Microbiol. 13:53-58.

Gelvin, S. B. 2010b. Plant proteins involved in Agrobacterium-mediated genetic transformation. Annu. Rev. Phytopathol. 48:45-68.

Gelvin, S. B., and Kim, S. I. 2007. Effect of chromatin upon Agrobacterium T-DNA integration and transgene expression. Biochim. Biophys. Acta 1769:410-421.

Goodrich, J., Puangsomlee, P., Martin, M., Long, D., Meyerowitz, E. M., and Coupland, G. 1997. A Polycomb-group gene regulates homeotic gene expression in Arabidopsis. Nature 386:44-51.

Grewal, S. I., and Jia, S. 2007. Heterochromatin revisited. Nat. Rev. Genet. 8:35-46.

Hecht, A., Laroche, T., Strahl-Bolsinger, S., Gasser, S. M., and Grunstein, M. 1995. Histone H3 and H4 N-termini interact with SIR3 and SIR4 proteins: A molecular model for the formation of heterochromatin in yeast. Cell 80:583-592.

Herz, H. M., Morgan, M., Gao, X., Jackson, J., Rickels, R., Swanson, S. K., Florens, L., Washburn, M. P., Eissenberg, J. C., and Shilatifard, A. 2014 Histone H3 lysine-to-methionine mutants as a paradigm to study chromatin signaling. Science 345:1065-1070.

Hood, E. E., Gelvin, S. B., Melchers, L. S., and Hoekema, A. 1993. New Agrobacterium helper plasmids for gene transfer to plants. Transgenic Res. 2:208-218.

Hwang, H. H., and Gelvin, S.B. 2004. Plant proteins that interact with VirB2, the Agrobacterium tumefaciens pilin protein, mediate plant transformation. Plant Cell 16:3148-3167.

Jefferson, R. A., Kavanagh, T. A., and Bevan, M. W. 1987. GUS fusions: $\beta$-Glucuronidase as a sensitive and versatile gene fusion marker in higher plants. EMBO J. 6:3901-3907.

Karantzali, E., Schulz, H., Hummel, O., Hubner, N., Hatzopoulos, A., and Kretsovali, A. 2008. Histone deacetylase inhibition accelerates the early events of stem cell differentiation: Transcriptomic and epigenetic analysis. Genome Biol. 9:R65

Kim, S. I., Veena, and Gelvin, S. B. 2007. Genome-wide analysis of Agrobacterium T-DNA integration sites in the Arabidopsis genome generated under non-selective conditions. Plant J. 51:779-791.

Köhler, C., Hennig, L., Spillane, C., Pien, S., Gruissem, W., and Grossniklaus, U. 2003. The Polycomb-group protein MEDEA regulates seed development by controlling expression of the MADS-box gene PHERES1. Genes Dev. 17:1540-1553.

Köhler, C., Page, D. R., Gagliardini, V., and Grossniklaus, U. 2005. The Arabidopsis thaliana MEDEA Polycomb group protein controls expression of PHERES1 by parental imprinting. Nat. Genet. 37:28-30.

Kuzmichev, A., Nishioka, K., Erdjument-Bromage, H., Tempst, P., and Reinberg, D. 2002. Histone methyltransferase activity associated with a human multiprotein complex containing the enhancer of zeste protein Genes Dev. 16:2893-2905.

Lacroix, B., Loyter, A., and Citovsky, V. 2008. Association of the Agrobacterium T-DNA-protein complex with plant nucleosomes. Proc. Natl. Acad. Sci. U.S.A. 105:15429-15434

Lafos, M., Kroll, P., Hohenstatt, M. L., Thorpe, F. L., Clarenz, O., and Schubert, D. 2011. Dynamic regulation of H3K27 trimethylation during Arabidopsis differentiation. PLoS Genet. 7:e1002040.

Lewis, P. W., Muller, M. M., Koletsky, M. S., Cordero, F., Lin, S., Banaszynski, L. A., Garcia, B. A., Muir, T. W., Becher, O. J., and Allis, C. D. 2013. Inhibition of PRC2 activity by a gain-of-function H3 mutation found in pediatric glioblastoma. Science 340:857-861

Li, M., Luo, J., Brooks, C. L., and Gu, W. 2002. Acetylation of p53 inhibits its ubiquitination by Mdm2. J. Biol. Chem. 277:50607-50611

Liu, C., Lu, F., Cui, X., and Cao, X. 2010. Histone methylation in higher plants. Annu. Rev. Plant Biol. 61:395-420.

Loidl, P. 2004. A plant dialect of the histone language. Trends Plant Sci. 9: 84-90.

Maison, C., Bailly, D., Peters, A. H., Quivy, J. P., Roche, D., Taddei, A., Lachner, M., Jenuwein, T., and Almouzni, G. 2002. Higher-order structure in pericentric heterochromatin involves a distinct pattern of histone modification and an RNA component. Nat. Genet. 30: 329-334.

Makarevich, G., Leroy, O., Akinci, U., Schubert, D., Clarenz, O., Goodrich, J., Grossniklaus, U., and Kohler, C. 2006. Different Polycomb group complexes regulate common target genes in Arabidopsis. EMBO Rep. 7: 947-952.

Martínez-Fernández, I., Sanchis, S., Marini, N., Balanza, V., Ballester, P., Navarrete-Gomez, M., Oliveira, A. C., Colombo, L., and Ferrandiz, C. 2014. The effect of NGATHA altered activity on auxin signaling pathways within the Arabidopsis gynoecium. Front. Plant Sci. 5:210

Mozgova, I., Kohler, C., and Hennig, L. 2015. Keeping the gate closed: Functions of the polycomb repressive complex PRC2 in development Plant J. 83:121-132.

Murashige, T., and Skoog, F. 1962. A revised medium for rapid growth and bio assays with tobacco tissue cultures. Physiol. Plant. 15:473-497.

Mysore, K. S., Nam, J., and Gelvin, S. B. 2000. An Arabidopsis histone H2A mutant is deficient in Agrobacterium T-DNA integration. Proc. Natl. Acad. Sci. U.S.A. 97:948-953.

Nakayama, J., Rice, J. C., Strahl, B. D., Allis, C. D., and Grewal, S. I. 2001. Role of histone H3 lysine 9 methylation in epigenetic control of heterochromatin assembly. Science 292:110-113.

Nam, J., Mysore, K. S., Zheng, C., Knue, M. K., Matthysse, A. G., and Gelvin, S. B. 1999. Identification of T-DNA tagged Arabidopsis mutants that are resistant to transformation by Agrobacterium. Mol. Gen. Genet. 261:429-438

Okada, T., Endo, M., Singh, M. B., and Bhalla, P. L. 2005. Analysis of the histone $\mathrm{H} 3$ gene family in Arabidopsis and identification of the malegamete-specific variant AtMGH3. Plant J. 44:557-568.

Page, R. D. 1996. TreeView: An application to display phylogenetic trees on personal computers. Comput. Appl. Biosci. 12:357-358.

Pasini, D., Malatesta, M., Jung, H. R., Walfridsson, J., Willer, A., Olsson, L. Skotte, J., Wutz, A., Porse, B., Jensen, O. N., and Helin, K. 2010 Characterization of an antagonistic switch between histone H3 lysine 27 methylation and acetylation in the transcriptional regulation of Polycomb group target genes. Nucleic Acids Res. 38:4958-4969.

Restrepo, M. A., Freed, D. D., and Carrington, J. C. 1990. Nuclear transport of plant potyviral proteins. Plant Cell 2:987-998.

Sardesai, N., Lee, L. Y., Chen, H., Yi, H., Olbricht, G. R., Stirnberg, A., Jeffries, J., Xiong, K., Doerge, R. W., and Gelvin, S. B. 2013. Cytokinins secreted by Agrobacterium promote transformation by repressing a plant myb transcription factor. Sci. Signal. 6:ra100.

Schmid, M., Davison, T. S., Henz, S. R., Pape, U. J., Demar, M., Vingron, M., Scholkopf, B., Weigel, D., and Lohmann, J. U. 2005. A gene expression map of Arabidopsis thaliana development. Nat. Genet. 37:501-506.

Schubert, D., Primavesi, L., Bishopp, A., Roberts, G., Doonan, J., Jenuwein, T., and Goodrich, J. 2006. Silencing by plant Polycombgroup genes requires dispersed trimethylation of histone $\mathrm{H} 3$ at lysine 27 . EMBO J. 25:4638-4649.

Schwartz, Y. B., Kahn, T. G., Stenberg, P., Ohno, K., Bourgon, R., and Pirrotta, V. 2010. Alternative epigenetic chromatin states of polycomb target genes. PLoS Genet. 6:e1000805.

Simon, J. A., and Kingston, R. E. 2013. Occupying chromatin: Polycomb mechanisms for getting to genomic targets, stopping transcriptional traffic, and staying put. Mol. Cell 49:808-824.

Tenea, G. N., Spantzel, J., Lee, L. Y., Zhu, Y., Lin, K., Johnson, S. J., and Gelvin, S. B. 2009. Overexpression of several Arabidopsis histone genes 
increases Agrobacterium-mediated transformation and transgene expression in plants. Plant Cell 21:3350-3367.

Thompson, J. D., Higgins, D. G., and Gibson, T. J. 1994. CLUSTAL W: Improving the sensitivity of progressive multiple sequence alignment through sequence weighting, position-specific gap penalties and weight matrix choice. Nucleic Acids Res. 22:4673-4680.

Tie, F., Banerjee, R., Stratton, C. A., Prasad-Sinha, J., Stepanik, V., Zlobin, A., Diaz, M. O., Scacheri, P. C., and Harte, P. J. 2009. CBP-mediated acetylation of histone H3 lysine 27 antagonizes Drosophila Polycomb silencing. Development 136:3131-3141.

Wu, G., Broniscer, A., McEachron, T. A., Lu, C., Paugh, B. S., Becksfort, J., Qu, C., Ding, L., Huether, R., Parker, M., Zhang, J., Gajjar, A., Dyer, M. A., Mullighan, C. G., Gilbertson, R. J., Mardis, E. R., Wilson, R. K., Downing, J. R., Ellison, D. W., Zhang, J., and Baker, S. J. 2012. Somatic histone $\mathrm{H} 3$ alterations in pediatric diffuse intrinsic pontine gliomas and non-brainstem glioblastomas. Nat. Genet. 44:251-253.

Xiang, C., Han, P., Lutziger, I., Wang, K., and Oliver, D. J. 1999. A mini binary vector series for plant transformation. Plant Mol. Biol. 40: 711-717.

Yi, H., Mysore, K. S., and Gelvin, S. B. 2002. Expression of the Arabidopsis histone H2A-1 gene correlates with susceptibility to Agrobacterium transformation. Plant J. 32:285-298.

Yi, H., Sardesai, N., Fujinuma, T., Chan, C. W., Veena, and Gelvin, S. B. 2006. Constitutive expression exposes functional redundancy between the Arabidopsis histone H2A gene HTA1 and other H2A gene family members. Plant Cell 18:1575-1589.
Zhang, H., Bishop, B., Ringenberg, W., Muir, W. M., and Ogas, J. 2012. The CHD3 remodeler PICKLE associates with genes enriched for trimethylation of histone H3 lysine 27. Plant Physiol. 159:418-432.

Zhang, H., Wang, B., Duan, C. G., and Zhu, J. K. 2013. Chemical probes in plant epigenetics studies. Plant Sig. Behav. 8:e25364. Published online.

Zhang, X., Clarenz, O., Cokus, S., Bernatavichute, Y. V., Pellegrini, M. Goodrich, J., and Jacobsen, S. E. 2007. Whole-genome analysis of histone H3 lysine 27 trimethylation in Arabidopsis. PLoS Biol. 5:e129.

Zhang, Z., Mao, Y., Ha, S., Liu, W., Botella, J. R., and Zhu, J. K. 2016. A multiplex CRISPR/Cas9 platform for fast and efficient editing of multiple genes in Arabidopsis. Plant Cell Rep. 35:1519-1533.

Zhu, Y., Nam, J., Humara, J. M., Mysore, K. S., Lee, L. Y., Cao, H., Valentine, L., Li, J., Kaiser, A. D., Kopecky, A. L., Hwang, H.-H., Bhattacharjee, S., Rao, P. K., Tzfira, T., Rajagopal, J., Yi, H., Veena, Yadav, B. S., Crane, Y. M., Lin, K., Larcher, Y., Gelvin, M. J. K., Knue, M., Ramos-Oliva, C., Zhao, X., Davis, S. J., Kim, S.-I., Ranjith-Kumar, C. T., Choi, Y.-J., Hallan, V. K., Chattopadhyay, S., Sui, X., Ziemienowicz, A., Matthysse, A. G., Citovsky, V., Hohn, B., and Gelvin, S. B. 2003. Identification of Arabidopsis rat mutants. Plant Physiol. 132: 494-505.

\section{AUTHOR-RECOMMENDED INTERNET RESOURCE}

The Arabidopsis Information Resource TAIR10 database: http://www.arabidopsis.org 